\title{
Final Study Report
}

National Cancer Institute

\section{Source}

National Cancer Institute. Final Study Report. NCI Thesaurus. Code C158361.

A report that is conclusive in its process or progression and which has been signed by the study director. 